PROCEEDINGS OF THE

AMERICAN MATHEMATICAL SOCIETY

Volume 138, Number 4, April 2010, Pages 1333-134

S 0002-9939(09)10112-0

Article electronically published on November 23, 2009

\title{
AN ENDPOINT ESTIMATE FOR THE CONE MULTIPLIER
}

\author{
YARYONG HEO, SUNGGEUM HONG, AND CHAN WOO YANG
}

(Communicated by Michael T. Lacey)

\begin{abstract}
In this paper we consider an endpoint estimate for high-dimensional cone multipliers.
\end{abstract}

\section{IntroduCtion AND STATEMENTS OF THE RESULTS}

Let $\widehat{\psi} \in C_{0}^{\infty}(\mathbb{R})$ be real-valued and supported in $\{1<\tau<2\}$. Then for each $\delta>0$, we consider the convolution operators $\mathrm{T}^{\delta}$ associated with the smooth cone multipliers given by

$$
\widehat{\mathrm{T}^{\delta} f}(\xi, \tau)=\widehat{f}(\xi, \tau)\left(1-\frac{|\xi|^{2}}{|\tau|^{2}}\right)_{+}^{\delta} \widehat{\psi}(\tau), \quad(\xi, \tau) \in \mathbb{R}^{d} \times \mathbb{R} .
$$

In the case $\delta>(d-1) / 2$, the convolution kernel belongs to $L^{1}$; hence $\mathrm{T}^{\delta}$ is an $L^{p}$ bounded operator for $1 \leq p \leq \infty$. In the case $0<\delta<(d-1) / 2$, it is conjectured that $\mathrm{T}^{\delta}$ is an $L^{p}$-bounded operator for

$$
\delta>\delta(p):=d|1 / p-1 / 2|-1 / 2, \quad 1<p<\infty .
$$

This conjectured range is the same as for the $d$-dimensional Bochner-Riesz multiplier problem. Note that the cone multiplier problem implies the Bochner-Riesz multiplier problem. By now the Bochner-Riesz multiplier problem is understood in the range $p<(2 d+4) /(d+4), d \geq 2$ (see [8]). But compared to the BochnerRiesz multiplier, little is known about the cone multiplier, and this conjecture still remains open for any $d \geq 2$. There are some partial results for this conjecture (see [3, 4, 5, 6, 7, 10]). In particular, in [10, the first author proved that $\mathrm{T}^{\delta}$ is an $L^{p}$-bounded operator for $1 \leq p \leq 2(d-1) /(d+1), \delta>\delta(p)$ and $d \geq 4$. This is the most recent result for high-dimensional cone multipliers. In this paper we consider an endpoint case $\delta=\delta(1)=(d-1) / 2$.

Theorem 1.1. If $d \geq 4$, then $\mathrm{T}^{(d-1) / 2}$ maps $L^{1}\left(\mathbb{R}^{d+1}\right)$ to $L^{1, \infty}\left(\mathbb{R}^{d+1}\right)$.

Received by the editors October 6, 2008, and, in revised form, July 8, 2009.

2000 Mathematics Subject Classification. Primary 42B15.

Key words and phrases. Cone multipliers.

The first author was supported by the Korea Research Foundation Grant funded by the Korean Government (KRF-2008-357-C00002).

The second author was supported by the Korea Research Foundation Grant funded by the Korean Government (MEST) (No. 2009-0065011).

The third author was supported by the Korea Research Foundation Grant funded by the Korean Government (KRF-2008-331-C00016). 
In the case of the Bochner-Riesz means, this result is well known for any dimension $d \geq 2$ (see [1, 2]). But for the cone multiplier there are some additional difficulties; i.e., in the case of the Bochner-Riesz multiplier, the main contribution of the convolution kernel comes from

$$
|x|^{-\delta-\frac{d+1}{2}} \mathrm{e}^{ \pm 2 \pi \mathrm{i}|x|}, \quad|x|>1, \quad x \in \mathbb{R}^{d},
$$

and in the case of the cone multiplier, the main contribution comes from

$$
|x|^{-\delta-\frac{d+1}{2}} \psi(t \pm|x|), \quad|x|>1, \quad(x, t) \in \mathbb{R}^{d} \times \mathbb{R} .
$$

As we can see in Lemma 2.3 and [1, 2], the term $\psi(t \pm|x|)$ in (1.2) plays a similar role as $\mathrm{e}^{ \pm 2 \pi \mathrm{i}|x|}$ in (1.1). But the convolution kernel (1.2) of the cone multiplier is defined on $\mathbb{R}^{d} \times \mathbb{R}$. This makes the cone multiplier problem more difficult than the Bochner-Riesz problem. Even though (1.2) is defined on $\mathbb{R}^{d} \times \mathbb{R}$, we can see that it is essentially supported in the cone $|t \pm| x|| \leq 1$, and we can use this advantage together with M. Christ's stopping time arguments (Lemma 2.2).

Remark 1.2. Recently, the second author, F. Nazarov and A. Seeger [12] obtained $L^{p}\left(\mathbb{R}^{d+1}\right) \rightarrow L^{p, \infty}\left(\mathbb{R}^{d+1}\right)$ inequalities for the cone multiplier $\left(1-|\xi|^{2} / \tau^{2}\right)_{+}^{\delta(p)}$, $(\xi, \tau) \in \mathbb{R}^{d} \times \mathbb{R}$ with $1<p<2(d-1) /(d+1)$ and $d \geq 4$. See also [1] for the improvements upon the existing results in the so-called local smoothing problem for the wave equation in high dimensions.

\section{Reductions And the PRoOf of TheOREm 1.1}

Notation. If $q$ is a dyadic cube in $\mathbb{R}^{d+1}$ with side-length $2^{j}$, we write $\ell(q)=j$. For each $j \in \mathbb{Z}, \mathbf{D}_{j}$ denotes the collection of dyadic cubes $q \in \mathbb{R}^{d+1}$ with $\ell(q)=j$, and for each $q \in \mathbf{D}_{j}, 2 q$ denotes $q+\left[-2^{j}, 2^{j}\right]^{d+1}$. For two quantities $A$ and $B$ we shall write $A \lesssim B$ if $A \leq C B$ for some absolute positive constant $C$. Also we shall write $A \sim B$ if $A \lesssim B$ and $B \lesssim A$. The Lebesgue measure on $\mathbb{R}^{d+1}$ of a subset $E$ will be denoted by $|E|$.

We need to show that

$$
\left|\left\{(x, t):\left|\mathrm{T}^{(d-1) / 2} f(x, t)\right|>\alpha\right\}\right| \leq C \alpha^{-1}\|f\|_{1},
$$

for each $\alpha>0$. We may assume $f \geq 0$. Also by limiting arguments we may assume that $f \in L^{1}\left(\mathbb{R}^{d+1}\right)$ have the form of a finite sum

$$
f(x, t)=\sum_{q \in \mathbf{q}} \alpha_{q} \chi_{q}(x, t),
$$

where $\alpha_{q}>0$ and $\mathbf{q}$ is a finite, disjoint collection of dyadic cubes. Moreover if $\ell(q) \geq 0$, then by dividing $q$ as smaller dyadic subcubes we may assume $\ell(q)=\ell \ll 0$ for all $q \in \mathbf{q}$. Let us explain the limiting arguments. Let $f \in L^{1}\left(\mathbb{R}^{d+1}\right)$ with $f \geq 0$. Then there exists a sequence $\left\{f_{m}\right\}_{m=1}^{\infty}$ of functions such that each $f_{m}$ has the form as in (2.2) and $\lim _{m \rightarrow \infty}\left\|f_{m}-f\right\|_{1}=0$. Also by choosing an appropriate subsequence we may assume

$$
\left\|f_{m}\right\|_{1} \leq 2\|f\|_{1}, \quad\left\|f_{m+1}-f_{m}\right\|_{1} \leq 2^{-2 m}\|f\|_{1} \quad \text { for each } m \text {. }
$$


Note that

$$
\begin{aligned}
\mathrm{T}^{\frac{d-1}{2}} f(x, t) & =\lim _{m \rightarrow \infty} \mathrm{T}^{\frac{d-1}{2}} f_{m}(x, t) \\
& =\mathrm{T}^{\frac{d-1}{2}} f_{1}(x, t)+\sum_{m=1}^{\infty} \mathrm{T}^{\frac{d-1}{2}}\left(f_{m+1}-f_{m}\right)(x, t)
\end{aligned}
$$

for almost every $(x, t)$. Hence if we prove (2.1) under the condition (2.2), then

$$
\begin{aligned}
& \left|\left\{\left|\mathrm{T}^{\frac{d-1}{2}} f(x, t)\right|>2 \alpha\right\}\right| \\
& \leq\left|\left\{\left|\mathrm{T}^{\frac{d-1}{2}} f_{1}(x, t)\right|>\alpha\right\}\right|+\sum_{m=1}^{\infty}\left|\left\{\left|\mathrm{T}^{\frac{d-1}{2}}\left(f_{m+1}-f_{m}\right)(x, t)\right|>2^{-m} \alpha\right\}\right| \\
& \leq C \alpha^{-1}\left\|f_{1}\right\|_{1}+\sum_{m=1}^{\infty} C\left(2^{-m} \alpha\right)^{-1}\left\|f_{m+1}-f_{m}\right\|_{1} \\
& \leq C \alpha^{-1}\|f\|_{1} .
\end{aligned}
$$

Therefore, from now on we assume that

$$
f=\sum_{q \in \mathbf{q}} \alpha_{q}|q| \frac{\chi_{q}}{|q|}=\sum_{q \in \mathbf{q}} \lambda_{q} a_{q}
$$

where $\lambda_{q}=\alpha_{q}|q|, a_{q}=\chi_{q} /|q|$ and $\mathbf{q}$ is a finite, disjoint collection of dyadic cubes in $\mathbf{D}_{\ell}$ for some $\ell \ll 0$.

Next we compute the inverse Fourier transform $K^{\delta}(x, t)$ of $\left(1-|\xi / \tau|^{2}\right)_{+}^{\delta} \widehat{\psi}(\tau)$. First note that

$$
K^{\delta}(x, t)=\int_{\mathbb{R}^{d}} \int_{\mathbb{R}} \mathrm{e}^{2 \pi \mathrm{i} \tau(x \cdot \xi+t)}\left(1-|\xi|^{2}\right)_{+}^{\delta} \tau^{d} \widehat{\psi}(\tau) d \tau d \xi,
$$

and by integration by parts via

$$
\frac{\partial}{\partial \tau} \mathrm{e}^{2 \pi \mathrm{i} \tau(x \cdot \xi+t)}=2 \pi \mathrm{i}(x \cdot \xi+t) \mathrm{e}^{2 \pi \mathrm{i} \tau(x \cdot \xi+t)},
$$

we have

$$
\left|K^{\delta}(x, t)\right| \leq C_{N} \sup _{|\xi| \leq 1}(1+|x \cdot \xi+t|)^{-N} \quad \text { for each } N>0 .
$$

From this, it is easy to see that $K^{\delta}(x, t) \chi_{(|x| \lesssim 1)} \in L^{1}\left(\mathbb{R}^{d+1}\right)$. Therefore, from now on, we assume $|x| \gtrsim 1$. The inverse Fourier transform of the Bochner-Riesz multiplier $\left(1-|\xi|^{2}\right)_{+}^{\delta}, \xi \in \mathbb{R}^{d}$ is given by

$$
\pi^{-\delta} \Gamma(1+\delta)|x|^{-\frac{d}{2}-\delta} J_{d / 2+\delta}(2 \pi|x|),
$$

where $J_{d / 2+\delta}$ is the Bessel function of order $d / 2+\delta$. So the inverse Fourier transform $K^{\delta}(x, t)$ of $\left(1-|\xi / \tau|^{2}\right)_{+}^{\delta} \widehat{\psi}(\tau)$ is given by

$$
K^{\delta}(x, t)=\int_{\mathbb{R}} \mathrm{e}^{2 \pi \mathrm{i} \tau t}\left(\pi^{-\delta} \Gamma(1+\delta)|\tau x|^{-\delta-d / 2} J_{d / 2+\delta}(2 \pi \tau|x|)\right) \tau^{d} \widehat{\psi}(\tau) d \tau .
$$


It is well-known that, for all nonnegative integers $N$ and $n$, as $r \rightarrow \infty$,

$$
\begin{gathered}
J_{m}(r)=\mathrm{e}^{\mathrm{i} r}\left[r^{-1 / 2} \sum_{j=0}^{N} a_{j} r^{-j / 2}+A_{N}(r)\right]+\mathrm{e}^{-\mathrm{i} r}\left[r^{-1 / 2} \sum_{j=0}^{N} b_{j} r^{-j / 2}+B_{N}(r)\right], \\
\frac{d^{n}}{d r^{n}} A_{N}(r)=O\left(r^{-n-\frac{N+1}{2}}\right), \quad \frac{d^{n}}{d r^{n}} B_{N}(r)=O\left(r^{-n-\frac{N+1}{2}}\right) .
\end{gathered}
$$

For reference, see pages $334-338$ in Stein's book 13. Therefore from the asymptotic expansion of the Bessel function $J_{d / 2+\delta}$, for any positive integers $N$ and $M$ we have

$$
K^{\delta}(x, t)=\sum_{j=0}^{N} \alpha_{j}^{ \pm}|x|^{-\left(\delta+\frac{d+1+j}{2}\right)} \mathcal{F}^{-1}\left[\tau^{-\delta+\frac{d-1-j}{2}} \widehat{\psi}(\tau)\right](t \pm|x|)+F_{N}^{\delta}(x, t),
$$

as $|x| \rightarrow \infty$; here $\mathcal{F}^{-1}$ denotes the inverse Fourier transform and

$$
\left|F_{N}^{\delta}(x, t)\right| \leq C_{\delta, N, M}|x|^{-\delta-\frac{d+1+N}{2}}(1+|| t|-| x||)^{-M} .
$$

Note that

$$
F_{N}^{(d-1) / 2} \chi_{(|x| \geq 1)} \in L^{1}\left(\mathbb{R}^{d+1}\right) \quad \text { if } N \geq 1 \text { and } M>1 .
$$

Therefore it suffices to consider the terms

$$
|x|^{-\delta-\frac{d+1+j}{2}} \mathcal{F}^{-1}\left[\tau^{-\delta+\frac{d-1-j}{2}} \widehat{\psi}(\tau)\right](t \pm|x|), \quad|x| \geq 1,
$$

with $j=0,1$, and $\delta=(d-1) / 2$. From now on we will concentrate on the term

$$
|x|^{-\delta-\frac{d+1}{2}} \mathcal{F}^{-1}\left[\tau^{-\delta+\frac{d-1}{2}} \widehat{\psi}(\tau)\right](t-|x|), \quad|x| \geq 1, \delta=(d-1) / 2,
$$

and the other cases can be treated similarly. Now, we should treat the operation of convolution with

$$
|x|^{-d} \psi(t-|x|), \quad|x|>1 .
$$

For technical reasons, to obtain the convolution estimates in Lemma2.3, fix a finite $C^{\infty}$ partition of unity $\left\{\omega_{i}\right\}$ on the unit sphere $\mathbb{S}^{d-1}$, with each $\omega_{i}$ having very small support. Let $\omega$ be one of the $C^{\infty}$ partition of unity $\left\{\omega_{i}\right\}$. Next choose $\eta \in C_{0}^{\infty}\left(\mathbb{R}^{d}\right)$, real-valued, radial and supported in $\{1 / 2 \leq|x| \leq 2\}$, so that $\sum_{j \in \mathbb{Z}} \eta\left(2^{-j} x\right)=1$ on $\mathbb{R}^{d} \backslash\{0\}$. Also choose $\phi \in C_{0}^{\infty}(\mathbb{R})$, real-valued and supported in $\{|t| \leq 2\}$, so that

$$
\sum_{n \in \mathbb{Z}} \phi(t-n):=\sum_{n \in \mathbb{Z}} \phi_{n}(t)=1
$$

Then it suffices to treat the operation of convolution with

$$
\sum_{n \in \mathbb{Z}} \sum_{j \geq 0}|x|^{-d} \psi(t-|x|) \omega(x /|x|) \phi(t-|x|-n) \eta\left(2^{-j} x\right):=\sum_{n \in \mathbb{Z}} \sum_{j \geq 0} K_{j}^{n}(x, t) .
$$

Let $f$ be as in (2.3). Then it suffices to show that

$$
\left|\left\{(x, t):\left|\left(\sum_{j \geq 0} K_{j}^{n}\right) * f(x, t)\right|>\alpha\right\}\right| \leq C_{N}(1+|n|)^{-N} \alpha^{-1}\|f\|_{1},
$$

for each $n \in \mathbb{Z}$ and $\alpha>0$. The following Lemma 2.1 is the standard CalderónZygmund decomposition, and we omit the proof (see Lemma 4.1 in [2]).

Lemma 2.1. Suppose $\beta>0$ is given. Then for any finite collection $\mathbf{q}$ of dyadic cubes $q$ and associated positive scalars $\lambda_{q}$, there exists a collection of pairwise 
disjoint dyadic cubes $\{S: S \in \mathbf{S}\}$ such that

(1) $\sum_{q: q \subset S} \lambda_{q} \leq 2^{d+1} \beta|S|$,

(2) $\sum_{S: S \in \mathbf{S}}|S| \leq \beta^{-1} \sum_{q \in \mathbf{q}} \lambda_{q}$

(3) $\left\|\sum_{\substack{q: \text { not contained } \\ \text { in any } S}} \frac{\lambda_{q}}{|q|} \chi_{q}\right\|_{\infty} \leq \beta$.

Let $\mathbf{C}$ be the collection of dyadic cubes $q \in \mathbf{q}$ which are contained in some $S \in \mathbf{S}$. For each $q \in \mathbf{C}$ we define $S(q)$ as the unique $S \in \mathbf{S}$ containing $q$. The following is a refined Calderón-Zygmund decomposition whose proof relies on a stopping time argument.

Lemma 2.2 (cf. Lemma 5.1 in [2] or Lemma 5 in [9]). Given $\beta>0$ there exists a function $\Gamma: \mathbf{C} \rightarrow \mathbb{Z}$ and a measurable set $E$ such that

(1) $|E| \leq C\left(\beta^{-1} \sum_{q \in \mathbf{C}} \lambda_{q}+\sum_{S \in \mathbf{S}}|S|\right)$,

(2) $\left\{q+\operatorname{supp}\left(K_{j}^{n}\right)\right\} \subset E$ for all $j<\Gamma(q)$ and $q \in \mathbf{C}$,

(3) $\ell(S(q))<\Gamma(q)$ for each $q \in \mathbf{C}$,

(4) for each $\tau, \ell \in \mathbb{Z}$ with $\ell \leq \tau$, and any $Q \in \mathbf{D}_{\ell}$ we have

$$
\sum_{q \subset Q, q \in \mathbf{C}, \Gamma(q) \leq \tau} \lambda_{q} \leq \beta 2^{d(\tau+1)+\max (0, \ell)} .
$$

For the proof we use the usual two-parameter stopping time arguments, and we construct an exceptional set $E$ by combining stopping time arguments with the support condition of the kernel $K_{j}^{n}$; i.e., if $\ell(Q) \leq \tau$ and $\tau \geq 0$, then

$$
\left|\bigcup_{j=0}^{\tau}\left(Q+\operatorname{supp}\left(K_{j}^{n}\right)\right)\right| \leq C 2^{d \tau+\max (0, \ell(Q))} .
$$

If $S \in \mathbf{S}$ has side-length $2^{j}$, then (1) of Lemma 2.1 says that

$$
\sum_{q: q \subset S} \lambda_{q} \leq 2^{d+1} \beta|S|
$$

But if $Q \subset S$, then by (4) of Lemma 2.2 we have a more delicate estimate:

$$
\sum_{q \subset Q, q \in \mathbf{C}, \Gamma(q) \leq j} \lambda_{q} \leq 2^{d} \beta 2^{j d+\max (0, \ell(Q))} .
$$

This is why we referred to Lemma 2.2 as a refined Calderón-Zygmund decomposition. The proof will be given in Section 4

Lemma 2.3. Let $\widetilde{K}_{j}^{n}$ denote the conjugate of $K_{j}^{n}$. Then for each $i<j$ and every $N>0$ we have

(1) $\left|K_{j}^{n} * \widetilde{K}_{j}^{n}(x, t)\right| \leq C_{N}(1+|n|)^{-2 N} 2^{-d j}(1+|(x, t)|)^{\frac{1-d}{2}} \chi_{\left(|(x, t)| \leq 2^{j+4}\right)}(x, t)$,

(2) $\left|K_{j}^{n} * \widetilde{K}_{i}^{n}(x, t)\right| \leq C_{N}(1+|n|)^{-2 N} 2^{-d j} 2^{\frac{1-d}{2} i} \chi_{\left(|x| \leq 2^{j+4}\right)}(x) \chi_{\left(|t-| x|| \leq 2^{i+4}\right)}(x, t)$. 
The proof of Lemma 2.3 will be given in Section 3. For the moment we assume Lemmas 2.2 and 2.3 and prove Theorem 1.1. Let $f$ be as in (2.3). We need to show that

$$
\left|\left\{(x, t):\left|\left(\sum_{j \geq 0} K_{j}^{n}\right) * f(x, t)\right|>\alpha\right\}\right| \leq C_{N}(1+|n|)^{-N} \alpha^{-1} \sum \lambda_{q},
$$

for each $\alpha>0$. Apply Lemma 2.1 to the collection of dyadic cubes $q$ and associated $\lambda_{q}$ appearing in the definition of $f$ with $\beta=(1+|n|)^{N} \alpha$. Let $\mathbf{S}$ be as in Lemma 2.1 and define

$$
b=\sum_{S \in \mathbf{S}} \sum_{q \subset S} \lambda_{q} a_{q}, \quad g=f-b .
$$

Then $\|g\|_{\infty} \leq \beta$ and so by (1) of Lemma 2.3, for $d \geq 4$ we have

$$
\begin{aligned}
\left\|\left(\sum_{j} K_{j}^{n}\right) * g\right\|_{2} & \leq \sum_{j}\|g\|_{2}\left\|K_{j}^{n} * \widetilde{K}_{j}^{n}\right\|_{1}^{1 / 2} \\
& \leq\|g\|_{2} \sum_{j}\left(C_{N}(1+|n|)^{-N} 2^{-j(d-3) / 4}\right) \\
& \leq C_{N}(1+|n|)^{-N} \beta^{1 / 2}\|f\|_{1}^{1 / 2} \\
& \leq C_{N}(1+|n|)^{-N / 2} \alpha^{1 / 2}\|f\|_{1}^{1 / 2}
\end{aligned}
$$

Therefore by Tchebychev's inequality we have

$$
\begin{aligned}
\left|\left\{(x, t):\left|\left(\sum_{j} K_{j}^{n}\right) * g(x, t)\right|>\alpha\right\}\right| & \leq \alpha^{-2}\left\|\left(\sum_{j} K_{j}^{n}\right) * g\right\|_{2}^{2} \\
& \leq C_{N}(1+|n|)^{-N} \alpha^{-1}\|f\|_{1} .
\end{aligned}
$$

Let $\mathbf{S}$ be as above and $\mathbf{C}$ be the collection of $q$ 's appearing in the definition of $b$. Then apply Lemma 2.2 with S, C. By (1) of Lemma 2.2 we have an exceptional set $E$ such that

$$
|E| \leq C\left(\beta^{-1} \sum_{q \in \mathbf{C}} \lambda_{q}+\sum_{S \in \mathbf{S}}|S|\right) \leq C \beta^{-1}\|f\|_{1} \leq C(1+|n|)^{-N} \alpha^{-1}\|f\|_{1} .
$$

By (2.6) and (2.7), (2.5) will follow from

$$
\left|\left\{(x, t) \in \mathbb{R}^{d+1} \backslash E:\left|\left(\sum_{j} K_{j}^{n}\right) * b(x, t)\right|>\alpha\right\}\right| \leq C_{N}(1+|n|)^{-N} \alpha^{-1} \sum_{q} \lambda_{q} .
$$

By Tchebychev's inequality, (2.8) will follow from

$$
\left\|\left(\sum_{j} K_{j}^{n}\right) * b\right\|_{L^{2}\left(\mathbb{R}^{d+1} \backslash E\right)}^{2} \leq C_{N}(1+|n|)^{-N} \alpha \sum_{q} \lambda_{q} .
$$

By (2) of Lemma 2.2, for each $q \in \mathbf{C}, K_{j}^{n} * a_{q}$ is supported in $E$ unless $j \geq \Gamma(q)$. Thus we have

$$
\begin{aligned}
& \left\|\sum_{j} b * K_{j}^{n}\right\|_{L^{2}\left(\mathbb{R}^{d+1} \backslash E\right)} \\
& \leq\left\|\sum_{j}\left(\sum_{\Gamma(q) \leq j} \lambda_{q} a_{q}\right) * K_{j}^{n}\right\|_{2} \\
& \leq\left\|\sum_{j}\left(\sum_{\Gamma(q) \leq 0} \lambda_{q} a_{q}\right) * K_{j}^{n}\right\|_{2}+\left\|\sum_{j}\left(\sum_{0<\Gamma(q) \leq j} \lambda_{q} a_{q}\right) * K_{j}^{n}\right\|_{2}
\end{aligned}
$$


and

$$
\left\|\sum_{j}\left(\sum_{0<\Gamma(q) \leq j} \lambda_{q} a_{q}\right) * K_{j}^{n}\right\|_{2}=\left\|\sum_{j} \sum_{0 \leq s<j}\left(\sum_{\Gamma(q)=j-s} \lambda_{q} a_{q}\right) * K_{j}^{n}\right\|_{2} .
$$

Note that

$$
\sum_{j} \sum_{0 \leq s<j}=\sum_{s \geq 0} \sum_{j>s}
$$

hence we have

$$
\left\|\sum_{j} \sum_{0 \leq s<j}\left(\sum_{\Gamma(q)=j-s} \lambda_{q} a_{q}\right) * K_{j}^{n}\right\|_{2} \leq \sum_{s \geq 0}\left\|\sum_{j>s}\left(\sum_{\Gamma(q)=j-s} \lambda_{q} a_{q}\right) * K_{j}^{n}\right\|_{2} .
$$

Now by (2.10) and (2.11) we have

$$
\left\|\sum_{j} b * K_{j}^{n}\right\|_{L^{2}\left(\mathbb{R}^{d+1} \backslash E\right)} \leq \digamma_{1}+\digamma_{2},
$$

where

$$
\digamma_{1}=\sum_{j}\left\|\left(\sum_{\Gamma(q) \leq 0} \lambda_{q} a_{q}\right) * K_{j}^{n}\right\|_{2}, \quad \digamma_{2}=\sum_{s \geq 0}\left\|\sum_{j>s}\left(\sum_{\Gamma(q)=j-s} \lambda_{q} a_{q}\right) * K_{j}^{n}\right\|_{2} .
$$

Estimation of part $\digamma_{1}$. Let $B_{0}=\sum_{\Gamma(q) \leq 0} \lambda_{q} a_{q}$. For each $q \in \mathbf{C}$ there exists a unique $S(q) \in \mathbf{S}$ containing $q$; hence if $\Gamma(q) \leq 0$, then by the condition $\ell(S(q)) \leq$ $\Gamma(q)$ we have $\ell(S(q)) \leq 0$. Therefore by (11) of Lemma 2.1, we can see that

$$
\left\|B_{0} \chi_{Q}\right\|_{1}=\sum_{q \subset Q, \Gamma(q) \leq 0} \lambda_{q} \leq C \beta \quad \text { for each } Q \in \mathbf{D}_{0} .
$$

By (11) of Lemma 2.3, we have

$$
\left\|K_{j}^{n} * \widetilde{K}_{j}^{n} * B_{0}\right\|_{\infty} \leq C_{N}(1+|n|)^{-2 N} 2^{-j d} \sup _{x \in \mathbb{R}^{d+1}}\left(\int_{|x-y| \lesssim 2^{j}} \frac{B_{0}(y)}{(1+|x-y|)^{\frac{d-1}{2}}} d y\right) .
$$

By (2.12) it is easy to see that

$$
\begin{aligned}
\left(\int_{|x-y| \lesssim 2^{j}} \frac{B_{0}(y)}{(1+|x-y|)^{\frac{d-1}{2}}} d y\right) & \leq\left(\int_{|x-y| \lesssim 2^{j}} \frac{C \beta}{(1+|x-y|)^{\frac{d-1}{2}}} d y\right) \\
& \leq C \beta 2^{\frac{d+3}{2} j} .
\end{aligned}
$$

Therefore we have

$$
\left\|K_{j}^{n} * \widetilde{K}_{j}^{n} * B_{0}\right\|_{\infty} \leq C_{N}(1+|n|)^{-2 N} \beta 2^{\frac{3-d}{2} j},
$$

and so for $d \geq 4$,

$$
\begin{aligned}
\digamma_{1}=\sum_{j}\left\|B_{0} * K_{j}^{n}\right\|_{2} & =\sum_{j}\left|\left\langle B_{0}, K_{j}^{n} * \widetilde{K}_{j}^{n} * B_{0}\right\rangle\right|^{1 / 2} \\
& \leq\left\|B_{0}\right\|_{1}^{1 / 2} \sum_{j}\left\|K_{j}^{n} * \widetilde{K}_{j}^{n} * B_{0}\right\|_{\infty}^{1 / 2} \\
& \leq C_{N}(1+|n|)^{-N} \beta^{1 / 2}\|f\|_{1}^{1 / 2} .
\end{aligned}
$$


Estimation of part $\digamma_{2}$. For each $l>0$, let $B_{l}=\sum_{\Gamma(q)=l} \lambda_{q} a_{q}$. Then

$$
\begin{aligned}
\left\|\sum_{j>s} B_{j-s} * K_{j}^{n}\right\|_{2}^{2} \leq & \sum_{j>s}\left\|B_{j-s} * K_{j}^{n}\right\|_{2}^{2} \\
& +2 \sum_{j>s}\left|\left\langle B_{j-s} * K_{j}^{n}, B_{j-1-s} * K_{j-1}^{n}\right\rangle\right| \\
& +2 \sum_{j>s} \sum_{s<i<j-1}\left|\left\langle B_{i-s} * K_{i}^{n} * \widetilde{K}_{j}^{n}, B_{j-s}\right\rangle\right| \\
:= & A_{1}(s)+A_{2}(s)+A_{3}(s) .
\end{aligned}
$$

For the part $A_{1}(s)$, we write

$$
\begin{aligned}
\left\|B_{j-s} * K_{j}^{n}\right\|_{2}^{2} & =\sum_{\Gamma(q), \Gamma\left(q^{\prime}\right)=j-s} \lambda_{q} \lambda_{q^{\prime}}\left\langle a_{q^{\prime}} * K_{j}^{n}, a_{q} * K_{j}^{n}\right\rangle \\
& \leq \sum_{m=0}^{j+4} \sum_{\substack{\Gamma(q), \Gamma\left(q^{\prime}\right)=j-s \\
1+\operatorname{dist}\left(q, q^{\prime}\right) \sim 2^{m}}} \lambda_{q} \lambda_{q^{\prime}}\left|\left\langle a_{q^{\prime}} * K_{j}^{n} * \widetilde{K}_{j}^{n}, a_{q}\right\rangle\right| \\
& \leq \sum_{m=0}^{j-s+2} \sum_{\substack{\Gamma(q), \Gamma\left(q^{\prime}\right)=j-s \\
1+\operatorname{dist}\left(q, q^{\prime}\right) \sim 2^{m}}}+\sum_{m=j-s+3} \sum_{\substack{\Gamma(q), \Gamma\left(q^{\prime}\right)=j-s \\
1+\operatorname{dist}\left(q, q^{\prime}\right) \sim 2^{m}}}^{j+4} \\
& :=\mathrm{I}+\mathrm{II} .
\end{aligned}
$$

Estimation of part I. For each fixed $m$ and $q^{\prime}$ consider the contribution of all $\lambda_{q}$ over all $q$ so that $1+\operatorname{dist}\left(q, q^{\prime}\right) \sim 2^{m}$. All such $q$ are contained in the union of a fixed number of $\mathbf{D}_{m}$. Hence when $0 \leq m \leq j-s+2$, by (11) of Lemma 2.3 we have

$$
\mathrm{I} \leq C_{N}(1+|n|)^{-2 N} 2^{-j d} \sum_{q^{\prime}} \lambda_{q^{\prime}} \sum_{m=0}^{j-s+2} \sum_{q: 1+\operatorname{dist}\left(q, q^{\prime}\right) \sim 2^{m}} 2^{\frac{1-d}{2} m} \lambda_{q} .
$$

By (4) of Lemma 2.2 we have

$$
\sum_{q: 1+\operatorname{dist}\left(q, q^{\prime}\right) \sim 2^{m}, \Gamma(q)=j-s} \lambda_{q} \leq C \beta 2^{(j-s) d+m},
$$

and so for $d \geq 4$,

$$
\mathrm{I} \leq C_{N}(1+|n|)^{-2 N} 2^{-d s} \beta \sum_{\Gamma\left(q^{\prime}\right)=j-s} \lambda_{q^{\prime}}
$$

Estimation of part II. Next, consider all $q$ with $\operatorname{dist}\left(q, q^{\prime}\right) \sim 2^{m}$ and $j-s+$ $3 \leq m \leq j+4$. Recall that each $q \in \mathbf{C}$ is contained in some $S(q) \in \mathbf{S}$. Since $\ell(S(q))<\Gamma(q)=j-s$, we have $\operatorname{dist}\left(S(q), q^{\prime}\right) \sim 2^{m}$ and so

$$
\begin{aligned}
\mathrm{II} & \leq C_{N}(1+|n|)^{-2 N} 2^{-j d} \sum_{q^{\prime}} \lambda_{q^{\prime}} \sum_{m=j-s+3}^{j+4} \sum_{q: \operatorname{dist}\left(q, q^{\prime}\right) \sim 2^{m}} 2^{\frac{1-d}{2} m} \lambda_{q} \\
& \leq C_{N}(1+|n|)^{-2 N} 2^{-j d} \sum_{q^{\prime}} \lambda_{q^{\prime}} \sum_{\substack{S \in \mathbf{S} \\
2^{j-s+2} \leq \operatorname{dist}\left(S, q^{\prime}\right) \leq 2^{j+5}}} \sum_{q \subset S} \operatorname{dist}\left(S, q^{\prime}\right)^{\frac{1-d}{2}} \lambda_{q} .
\end{aligned}
$$


By (11) of Lemma 2.1, for each $S \in \mathbf{S}$ we have $\sum_{q \subset S} \lambda_{q} \leq C \beta|S|$, and so

$$
\begin{aligned}
\sum_{\substack{S \in \mathbf{S} \\
2^{j-s+2} \leq \operatorname{dist}\left(S, q^{\prime}\right) \leq 2^{j+5}}} \sum_{q \subset S} \operatorname{dist}\left(S, q^{\prime}\right)^{\frac{1-d}{2}} \lambda_{q} & \leq C \sum_{\substack{2^{j-s+2} \leq \operatorname{dist}\left(S, q^{\prime}\right) \leq 2^{j+5}\\
}} \operatorname{dist}\left(S, q^{\prime}\right)^{\frac{1-d}{2}}(\beta|S|) \\
& \leq C \beta \int_{y \in \mathbb{R}^{d+1}, 2^{j-s+2} \leq|y| \leq 2^{j+5}}|y|^{\frac{1-d}{2}} d y \\
& \leq C 2^{\frac{3-d}{2} j} \beta .
\end{aligned}
$$

Now we have

$$
\mathrm{II} \leq C_{N}(1+|n|)^{-2 N} 2^{\frac{3-d}{2} j} \beta \sum_{\Gamma\left(q^{\prime}\right)=j-s} \lambda_{q^{\prime}}
$$

and so for $d \geq 4$,

$$
\begin{aligned}
A_{1}(s) & \leq \sum_{j>s} C_{N}(1+|n|)^{-2 N}\left(2^{-s d}+2^{\frac{3-d}{2} j}\right) \beta \sum_{\Gamma\left(q^{\prime}\right)=j-s} \lambda_{q^{\prime}} \\
& \leq C_{N}(1+|n|)^{-2 N} 2^{\frac{3-d}{2} s} \beta \sum_{q^{\prime}} \lambda_{q^{\prime}} .
\end{aligned}
$$

Similarly we have

$$
A_{2}(s) \leq C_{N}(1+|n|)^{-2 N} 2^{\frac{3-d}{2} s} \beta \sum_{q^{\prime}} \lambda_{q^{\prime}} .
$$

For the part $A_{3}(s)$, if $\Gamma(q)=i-s$, then $\ell(q), S(q) \leq i-s$. So by (11) of Lemma 2.1 and (2) of Lemma (2.3) together with the condition

$$
\operatorname{supp}\left(K_{i}^{n} * \widetilde{K}_{j}^{n}\right) \subset\left\{(x, t):|x| \leq 2^{j+4},|t-| x|| \leq 2^{i+4}\right\}
$$

we have

$$
\begin{aligned}
\left\|B_{i-s} * K_{i}^{n} * \widetilde{K}_{j}^{n}\right\|_{\infty} & \leq C_{N}(1+|n|)^{-2 N} 2^{-j d} 2^{\frac{1-d}{2} i} \sum_{S \in \mathbf{S}} \sum_{\substack { q \subset S \\
\begin{subarray}{c}{q \\
(q)=i-s{ q \subset S \\
\begin{subarray} { c } { q \\
( q ) = i - s } }\end{subarray}} \lambda_{q} \\
& \leq C_{N}(1+|n|)^{-2 N} 2^{-j d} 2^{\frac{1-d}{2} i} \sum_{S \in \mathbf{S}} \beta|S|,
\end{aligned}
$$

where the sums are taken over all $S \in \mathbf{S}$ such that

$$
S \subset\left\{(x, t):|x| \leq 2^{j+4},|t-| x|| \leq 2^{i+4}\right\} .
$$

So we have $\sum_{S \in \mathbf{S}}|S| \leq C 2^{j d+i}$ and

$$
\left\|B_{i-s} * K_{i}^{n} * \widetilde{K}_{j}^{n}\right\|_{\infty} \leq C_{N}(1+|n|)^{-2 N} 2^{\frac{3-d}{2} i} \beta .
$$

Therefore for $d \geq 4$,

$$
\begin{aligned}
A_{3}(s) & =\sum_{j>s} \sum_{s<i<j-1}\left|\left\langle B_{i-s} * K_{i}^{n} * \widetilde{K}_{j}^{n}, B_{j-s}\right\rangle\right| \\
& \leq C_{N}(1+|n|)^{-2 N} \sum_{j>s} \sum_{i>s} 2^{\frac{3-d}{2} i} \beta\left\|B_{j-s}\right\|_{1} \\
& \leq C_{N}(1+|n|)^{-2 N} 2^{\frac{3-d}{2} s} \beta \sum_{q} \lambda_{q} .
\end{aligned}
$$


Finally from (2.13), (2.14), (2.15) and (2.16), for $d \geq 4$ we have

$$
\digamma_{2} \leq \sum_{s \geq 0}\left(A_{1}(s)+A_{2}(s)+A_{3}(s)\right)^{1 / 2} \leq C_{N}(1+|n|)^{-N} \beta^{1 / 2}\left(\sum_{q} \lambda_{q}\right)^{1 / 2},
$$

and we are done.

\section{Proof of Lemma 2.3}

The proof is similar to Lemma 3.1 in [2]. For (1), let $\eta_{1}(x):=\eta(x)|x|^{-d}$. Then we have

$$
K_{j}^{n} * \widetilde{K}_{j}^{n}(x, t)=2^{-2 d j} \int_{\mathbb{R}} \int_{0}^{\infty} F_{j}(x, t, s, r) \bar{\psi}(s-r) \eta_{1}\left(2^{-j} r\right) \phi_{n}(s-r) r^{d-1} d r d s,
$$

where

$$
\begin{array}{r}
F_{j}(x, t, s, r)=\int_{\mathbb{S}^{d-1}} \psi(t+s-|x+r \theta|) \omega[(x+r \theta) /|x+r \theta|] \omega(\theta) \\
\times \phi_{n}(t+s-|x+r \theta|) \eta_{1}\left[2^{-j}(x+r \theta)\right] d \theta .
\end{array}
$$

Note that

$$
\psi(t+s-|x+r \theta|)=\int_{\mathbb{R}} \mathrm{e}^{2 \pi \mathrm{i} \tau(t+s-|x+r \theta|)} \widehat{\psi}(\tau) d \tau .
$$

Hence we have

$$
F_{j}(x, t, s, r)=\int_{\mathbb{R}} \mathrm{e}^{2 \pi \mathrm{i} \tau(t+s)} \widehat{\psi}(\tau) G_{j}(x, t, s, r, \tau) d \tau,
$$

where

$$
\begin{aligned}
G_{j}(x, t, s, r, \tau)= & \int_{\mathbb{S}^{d-1}} \mathrm{e}^{-2 \pi \mathrm{i} \tau|x+r \theta|} \omega[(x+r \theta) /|x+r \theta|] \omega(\theta) \\
& \times \phi_{n}(t+s-|x+r \theta|) \eta_{1}\left[2^{-j}(x+r \theta)\right] d \theta .
\end{aligned}
$$

It suffices to show that

$$
\left|G_{j}(x, t, s, r, \tau)\right| \leq C(1+|(x, t)|)^{(1-d) / 2},
$$

uniformly for $\tau \sim 1, s$ and $r \sim 2^{j}$. Suppose that the function $\omega$ from the partition of unity has sufficiently small support about $(0, \ldots, 0,1)$. Then we use the local coordinate chart

$$
\theta=\left(\theta_{1}, \ldots, \theta_{d}\right)=\left(\theta_{1}, \ldots, \theta_{d-1}, \sqrt{1-\theta_{1}^{2}-\cdots-\theta_{d-1}^{2}}\right) .
$$

Then by direct calculation, for $1 \leq l \leq d-1$,

$$
\begin{gathered}
\frac{\partial}{\partial \theta_{l}}(|x+r \theta|)=r\left(\frac{\theta_{d} x_{l}-\theta_{l} x_{d}}{|x+r \theta| \theta_{d}}\right), \\
\frac{\partial^{2}|x+r \theta|}{\partial \theta_{l}^{2}}=r\left(\frac{|x+r \theta|^{2}\left(\theta_{d}^{2}-\theta_{l}^{2}\right)\left(-x_{d}\right)-r \theta_{d}\left|\theta_{d} x_{l}-\theta_{l} x_{d}\right|^{2}}{|x+r \theta|^{3} \theta_{d}^{3}}\right),
\end{gathered}
$$

and for $1 \leq l \neq m \leq d-1$,

$$
\frac{\partial^{2}|x+r \theta|}{\partial \theta_{l} \partial \theta_{m}}=-r\left(\frac{\theta_{l} \theta_{m} x_{d}|x+r \theta|^{2}+r \theta_{d}\left(\theta_{d} x_{m}-\theta_{m} x_{d}\right)\left(\theta_{d} x_{l}-\theta_{l} x_{d}\right)}{|x+r \theta|^{3} \theta_{d}^{3}}\right) .
$$

Note that

$$
\frac{\partial^{2}|x+r \theta|}{\partial \theta_{l}^{2}}(0, \ldots, 0,1)=r\left(\frac{|x+r \theta|^{2}\left(-x_{d}\right)-x_{l}^{2}}{|x+r \theta|^{3}}\right)
$$


and

$$
\frac{\partial^{2}|x+r \theta|}{\partial \theta_{l} \partial \theta_{m}}(0, \ldots, 0,1)=\frac{-r^{2} x_{l} x_{m}}{|x+r \theta|^{3}}, \quad 1 \leq l \neq m \leq d-1 .
$$

In the case $\left|x_{l}\right| \gtrsim|x|$ for some $1 \leq l \leq d-1$, by (3.3),

$$
\left|\frac{\partial|x+r \theta|}{\partial \theta_{l}}\right| \gtrsim\left|x_{l}\right|
$$

uniformly for $|x+r \theta| \sim 2^{j}, r \sim 2^{j}$. By integrating by parts via

$$
\frac{\partial}{\partial \theta_{l}}\left(\mathrm{e}^{-2 \pi \mathrm{i} \tau|x+r \theta|}\right)=-2 \pi \mathrm{i} \tau \frac{\partial|x+r \theta|}{\partial \theta_{l}}\left(\mathrm{e}^{-2 \pi \mathrm{i} \tau|x+r \theta|}\right)
$$

we have

$$
\left|G_{j}(x, t, s, r, \tau)\right| \leq C_{N}\left(1+\left|x_{l}\right|\right)^{-N} \leq C_{N}(1+|x|)^{-N} .
$$

In the case $\left|x_{d}\right| \gtrsim|x|$, from (3.4), (3.5), (3.6), (3.7), together with the conditions $|x+r \theta| \sim 2^{j}, r \sim 2^{j}$, we can see that each absolute value of the eigenvalue of the Hessian matrix

$$
\frac{\partial^{2}|x+r \theta|}{\partial^{2} \theta}
$$

is bounded below by $C\left|x_{d}\right|$ uniformly for $2^{j-1}<r<2^{j+1}$ when $\theta$ and $(x+r \theta) / \mid x+$ $r \theta \mid$ are both in the support of $\omega$. By the methods of stationary phase, we have

$$
\left|G_{j}(x, t, s, r, \tau)\right| \leq C\left(1+\left|x_{d}\right|\right)^{(1-d) / 2} \leq(1+|x|)^{(1-d) / 2} .
$$

Hence we have

$$
\left|K_{j}^{n} * \widetilde{K}_{j}^{n}(x, t)\right| \leq C_{N}(1+|n|)^{-2 N} 2^{-j d}(1+|x|)^{(1-d) / 2} .
$$

Next recall that

$$
\begin{array}{r}
K_{j}^{n} * \widetilde{K}_{j}^{n}(x, t)=2^{-2 d j} \int_{\mathbb{R}} \int_{\mathbb{R}^{d}} \psi(t+s-|x+y|) \bar{\psi}(s-|y|) \omega[(x+y) /|x+y|] \\
\quad \times \omega(y /|y|) \phi_{n}(t+s-|x+y|) \phi_{n}(s-|y|) \eta_{1}\left[2^{-j}(x+y)\right] \eta_{1}\left(2^{-j} y\right) d y d s .
\end{array}
$$

Hence $K_{j}^{n} * \widetilde{K}_{j}^{n}(x, t)$ is supported in

$$
|t+s-| x+y|-n| \leq 2, \quad|s-| y|-n| \leq 2,
$$

and by the triangle inequality, this implies that

$$
|t| \leq|t+s-| x+y|-n|+|-s+| y|+n|+|-| y|+| x-y|| \leq 2+2+|x| .
$$

Therefore if $|x| \leq 2$, then $|(x, t)| \leq 6$, and (3.8) implies that

$$
\left|K_{j}^{n} * \widetilde{K}_{j}^{n}(x, t)\right| \leq C_{N}(1+|n|)^{-2 N} 2^{-j d}(1+|(x, t)|)^{-N} .
$$

If $|x| \geq 2$, then by (3.9) we have $|t| \leq 3|x|$, and (3.8) implies that

$$
\left|K_{j}^{n} * \widetilde{K}_{j}^{n}(x, t)\right| \leq C_{N}(1+|n|)^{-2 N} 2^{-j d}(1+|(x, t)|)^{(1-d) / 2} .
$$

For (2), let $F_{j}(x, t, s, r)$ and $G_{j}(x, t, s, r, \tau)$ be the same as in (3.1) and (3.2). Then we have

$$
K_{j}^{n} * \widetilde{K}_{i}^{n}(x, t)=2^{-d j-d i} \int_{\mathbb{R}} \int_{0}^{\infty} F_{j}(x, t, s, r) \bar{\psi}(s-r) \eta_{1}\left(2^{-i} r\right) \phi_{n}(s-r) r^{d-1} d r d s .
$$


Since $|x+r \theta| \sim 2^{j}, r \sim 2^{i}$, we have $|x| \sim 2^{j}$. Hence in the case $\left|x_{l}\right| \gtrsim|x|$ for some $1 \leq l \leq d-1$, from (3.3) we can see that

$$
\left|\frac{\partial|x+r \theta|}{\partial \theta_{l}}\right| \gtrsim 2^{i}
$$

uniformly for $|x+r \theta| \sim 2^{j}$ and $r \sim 2^{i}$, and by integrating by parts via

$$
\frac{\partial}{\partial \theta_{l}}\left(\mathrm{e}^{-2 \pi \mathrm{i} \tau|x+r \theta|}\right)=-2 \pi \mathrm{i} \tau \frac{\partial|x+r \theta|}{\partial \theta_{l}}\left(\mathrm{e}^{-2 \pi \mathrm{i} \tau|x+r \theta|}\right)
$$

we have

$$
\left|G_{j}(x, t, s, r, \tau)\right| \leq C_{N} 2^{-N i} .
$$

In the case $\left|x_{d}\right| \gtrsim|x|$, each absolute value of the eigenvalue of the Hessian matrix

$$
\frac{\partial^{2}|x+r \theta|}{\partial^{2} \theta}
$$

is bounded below by $C 2^{i}$ uniformly for $r \sim 2^{i}$ when $\theta$ and $(x+r \theta) /|x+r \theta|$ are both in support of $\omega$. By the methods of stationary phase, we have

$$
\left|G_{j}(x, t, s, r, \tau)\right| \leq C 2^{\frac{1-d}{2} i} .
$$

Hence in the case $\left|x_{d}\right| \gtrsim|x|$ we have

$$
\left|K_{j}^{n} * \widetilde{K}_{i}^{n}(x, t)\right| \leq C_{N}(1+|n|)^{-2 N} 2^{-j d} 2^{\frac{1-d}{2} i} .
$$

Note that $K_{j}^{n} * \widetilde{K}_{i}^{n}(x, t)$ is supported in

$$
|t+s-| x+y|-n| \leq 2,|s-| y|-n| \leq 2,|x+y| \leq 2^{j+1},|y| \leq 2^{i+1} .
$$

Hence $K_{j}^{n} * \widetilde{K}_{i}^{n}(x, t)$ is supported in

$$
\begin{aligned}
|t-| x|| & \leq|t+s-| x+y|-n|+|| x+y|-| x||+|-s+n| \\
& \leq 2+|y|+(2+|y|) \leq 2^{i+4}
\end{aligned}
$$

and from (3.10) and (3.11) we have (2) of Lemma 2.3

\section{Proof of Lemma 2.2}

The following are the usual two-parameter stopping-time arguments. These will be discussed in more detail below. We will combine these arguments with the support condition of the kernel $K_{j}^{n}$ to construct an exceptional set $E$. Let $m=$ $\min \{\ell(q): q \in \mathbf{C}\}$. Select an integer $\tau_{0}$ such that

$$
\tau_{0}>\max \{\ell(q): q \in \mathbf{C}\}, \quad \sum_{q \in \mathbf{C}} \lambda_{q}<\beta 2^{d \tau_{0}+\max (0, m)} .
$$

For each fixed $\tau \in \mathbb{Z}$ with $\tau \leq \tau_{0}$, we will define a sequence of functions $\Lambda_{\tau, \ell}$ : $\mathbf{D}_{\ell} \rightarrow \mathbb{R}$ by a descending induction on $\ell \in \mathbb{Z}$ with $\ell \leq \tau$, and proceed with the same construction by a descending induction on $\tau$. At each step, we will define subsets $\mathbf{C}_{1}, \mathbf{C}_{2}$ of $\mathbf{C}$ which will increase as we proceed. Let $\mathbf{C}_{1}, \mathbf{C}_{2} \subset \mathbf{C}$ and $\tau \in \mathbb{Z}$ be fixed for the moment, and define Inner loop as 
Inner loop. Define $\Lambda_{\tau, \ell}: \mathbf{D}_{\ell} \rightarrow \mathbb{R}$ with $\ell \leq \tau$. For each $Q \in \mathbf{D}_{\ell}$, define

$$
\Lambda_{\tau, \ell}(Q)=\sum_{q \subset Q:} \lambda_{q \notin \mathbf{C}_{1} \cup \mathbf{C}_{2}} .
$$

Begin with $\ell=\tau$. If

$$
\Lambda_{\tau, \ell}(Q)>\beta 2^{d \tau+\max (0, \ell)},
$$

then we say that " $Q$ is selected at step $(\tau, \ell)$ ". Put into $\mathbf{C}_{1}$ every $q$ such that $q \subset Q$ and define $\Gamma(q)=1+\tau$. Repeat until $\ell<\min \{\ell(q): q \in \mathbf{C}\}$. Actually this part of the process will be terminated once $\ell$ is smaller than $m$. Put into $\mathbf{C}_{2}$ every $q \in \mathbf{C} \backslash \mathbf{C}_{1}$ such that $\ell(q) \geq \tau$ and for such $q$ define $\Gamma(q)=1+\ell(S(q))$. Actually every $q \in \mathbf{C} \backslash \mathbf{C}_{1} \cup \mathbf{C}_{2}$ satisfies $\ell(q) \leq \tau-1$.

Perform Inner loop with $\mathbf{C}_{1}=\emptyset=\mathbf{C}_{2}$ and $\tau=\tau_{0}$. Next replace $\tau$ by $\tau-1$ and repeat Inner loop. Repeat until $\tau=m-1$. After this process we obtain $\mathbf{C}=\mathbf{C}_{1} \cup \mathbf{C}_{2}$. Clearly all selected $Q$ are disjoint and $\Gamma$ is well defined. Note that there is the usual stopping-time condition

$$
\Lambda_{\tau, \ell}(Q) \leq \beta 2^{d(\tau+1)+\max (0, \ell)},
$$

which holds for all $Q \in \mathbf{D}_{\ell}$ when $\ell \leq \tau \leq \tau_{0}$. This is because if $\tau=\tau_{0}$, then the condition is clear from the choice of $\tau_{0}$ and $m$. When $\ell \leq \tau \leq \tau_{0}$, suppose this fails. Then $\Lambda_{\tau+1, \ell}(Q) \geq \Lambda_{\tau, \ell}(Q)>\beta 2^{d(\tau+1)+\max (0, \ell)}$. This means that $Q$ is selected at step $(\tau+1, \ell)$; hence $\Lambda_{\tau, \ell}(Q)=0$, and we have the contradiction.

Next we show (4), which says, for each $Q \in \mathbf{D}_{\ell}$ with $\ell \leq \tau$,

$$
\sum_{q \subset Q: \Gamma(q) \leq \tau} \lambda_{q} \leq \beta 2^{d(\tau+1)+\max (0, \ell)} .
$$

When $\tau \geq \tau_{0}$, this property is clear from the initial choice of $\tau_{0}$. When $\tau<\tau_{0}$, if we show that

$$
\Lambda_{\tau, \ell}(Q)=\sum_{q \subset Q: q \notin \mathbf{C}_{1} \cup \mathbf{C}_{2}} \lambda_{q} \geq \sum_{q \subset Q: \Gamma(q) \leq \tau} \lambda_{q}
$$

for each $Q \in \mathbf{D}_{\ell}$ with $\ell \leq \tau<\tau_{0}$, then by combining (4.2) and (4.3), we have (4). Now (4.3) follows from the definition

$$
\Lambda_{\tau, \ell}(Q)=\sum_{q \subset Q:} \lambda_{q \notin \mathbf{C}_{1} \cup \mathbf{C}_{2}}
$$

and the fact that

$$
\Gamma(q) \leq \tau \Rightarrow q \notin \mathbf{C}_{1} \cup \mathbf{C}_{2} \text { at the beginning of step }(\tau, \ell) .
$$

This is because, if $q \in \mathbf{C}_{1}$, then $\Gamma(q) \geq 1+\tau>\tau$, and if $q \in \mathbf{C}_{2}$, then $\Gamma(q)=$ $1+\ell(S(q)) \geq 1+(1+\tau)>\tau$. Hence $\Gamma(q) \leq \tau$ implies $q \notin \mathbf{C}_{1} \cup \mathbf{C}_{2}$, and so we have (4.3).

Next, we construct an exceptional set $E$ by using the above stopping-time arguments. If $Q$ is selected at step $(\tau, \ell)$, then we define $\tau(Q)=\tau$. If $\tau(Q) \geq 0$, then we define the tendril $T(Q)$ associated to $Q$ by

$$
T(Q)=\bigcup_{j<\tau(Q)+1}\left(Q+\operatorname{supp}\left(K_{j}^{n}\right)\right) .
$$


Also we define

$$
E=E_{1} \cup E_{2}, \quad E_{1}=\bigcup_{S \in \mathbf{S}} 2 S, \quad E_{2}=\bigcup_{Q: \text { selected }, \tau(Q) \geq 0} T(Q) .
$$

Thus we have

$$
\left|E_{1}\right| \leq C \sum_{S \in \mathbf{S}}|S| .
$$

By the measure condition (2.4) of $T(Q)$ and the condition (4.1) of selected $Q$,

$$
\begin{aligned}
\left|E_{2}\right| & \leq \sum_{Q: \text { selected }, \tau(Q) \geq 0}|T(Q)| \leq C \sum_{Q: \text { selected }} 2^{d \tau(Q)+\max (0, \ell(Q))} \\
& \leq C \frac{1}{\beta} \sum_{Q: \text { selected }} \Lambda_{\tau, \ell}(Q) \leq C \frac{1}{\beta} \sum \lambda_{q} .
\end{aligned}
$$

So we have (11). For (2), if $q \in \mathbf{C}_{1}$, then $q$ belongs to some selected $Q$ and $\Gamma(q)=$ $\tau(Q)+1$. Hence if $\Gamma(q)>j$, then

$$
\bigcup_{j<\Gamma(q)}\left(q+\operatorname{supp}\left(K_{j}^{n}\right)\right) \subset T(Q) \subset E_{2},
$$

and if $q \in \mathbf{C}_{2}$, then $q$ belongs to some $S(q) \in \mathbf{S}$ and $\Gamma(q)=1+\ell(S(q))$. Hence if $\Gamma(q)>j$, then

$$
\bigcup_{j<\Gamma(q)}\left\{q+\operatorname{supp}\left(K_{j}^{n}\right)\right\} \subset 2 S(q) \subset E_{1} .
$$

For (3), let $\Gamma^{\prime}:=\Gamma$ and redefine

$$
\Gamma(q)=\max \left\{\Gamma^{\prime}(q), 1+\ell(S(q))\right\} .
$$

Then (11) and (3) are satisfied. We must check (2) and (4). For (2), if $\Gamma(q)=\Gamma^{\prime}(q)$, then there is no problem. If $\Gamma(q)=1+\ell(S(q))>j$, then the argument is the same as above. (4) follows from

$$
\sum_{q \subset Q: \Gamma(q) \leq \tau} \lambda_{q} \leq \sum_{q \subset Q: \Gamma^{\prime}(q) \leq \tau} \lambda_{q},
$$

and we have Lemma 2.2

\section{REFERENCES}

1. M. Christ and C. D. Sogge, On the $L^{1}$ behavior of eigenfunction expansions and singular integral operators, in Miniconferences on harmonic analysis and operator algebras (Canberra, 1987), 29-50, Austral. Nat. Univ., Canberra, 1988. MR0953981 (89k:42024)

2. M. Christ, Weak type $(1,1)$ bounds for rough operators, Ann. of Math. (2) 128 (1988), no. 1, 19-42. MR0951506 (89m:42013)

3. G. Mockenhaupt, A note on the cone multiplier, Proc. Amer. Math. Soc. 117 (1993), no. 1, 145-152. MR1098404 (93c:42015)

4. G. Garrigós and A. Seeger, On plate decompositions of cone multipliers, Proceedings of the Conference on Harmonic Analysis and Its Applications, Hokkaido University, Sapporo (2005).

5. J. Bourgain, Estimates for cone multipliers, in Geometric aspects of functional analysis (Israel, 1992-1994), 41-60, Birkhäuser, Basel, 1995. MR1353448 (96m:42022)

6. T. Wolff, Local smoothing type estimates on $L^{p}$ for large p, Geom. Funct. Anal. 10 (2000), no. 5, 1237-1288. MR.1800068 (2001k:42030)

7. I. Łaba and T. Wolff, A local smoothing estimate in higher dimensions, J. Anal. Math. 88 (2002), 149-171. MR.1956533 (2005b:35015)

8. S. Lee, Improved bounds for Bochner-Riesz and maximal Bochner-Riesz operators, Duke Math. J. 122 (2004), no. 1, 205-232. MR2046812(2005e:42042) 
9. Y. R. Heo, An endpoint estimate for some maximal operators associated to submanifolds of low codimension, Pacific J. Math. 201 (2001), no. 2, 323-338. MR.1875897 (2002j:42030)

10. Y. Heo, Improved bounds for high dimensional cone multipliers, Indiana Univ. Math. J. 58 (2009), no. 3, 1187-1202. MR2541363

11. Y. Heo, F. Nazarov and A. Seeger, Radial Fourier multipliers in high dimensions, preprint.

12. Y. Heo, F. Nazarov and A. Seeger, On radial and conical Fourier multipliers, preprint.

13. E. M. Stein, Harmonic analysis: Real-variable methods, orthogonality, and oscillatory integrals, Princeton Univ. Press, Princeton, NJ, 1993. MR.1232192 (95c:42002)

Department of Mathematics, University of Wisconsin-Madison, Madison, Wisconsin 53706

E-mail address: heo@math.wisc.edu

Current address: Pohang Mathematics Institute, Pohang University of Science \& Technology, Pohang 790-784, Korea

E-mail address: heo@postech.ac.kr

Department of Mathematics, Chosun University, Ghanguu 501-759, Republic of KOREA

E-mail address: skhong@mail.chosun.ac.kr

Department of Mathematics, Korea University, Seoul 136-701, Republic of Korea

E-mail address: cw_yang@korea.ac.kr 